\title{
Theoretical and experimental study of 3-D initial fracture and its significance to faulting*
}

\author{
Shiyu Li ${ }^{1, \star} \quad$ Taiming He ${ }^{1}$ Chunkai Teng ${ }^{2}$ \\ Xuyao Zheng ${ }^{1}$ and Kuksenko Viktor ${ }^{3}$ \\ ${ }^{1}$ Institute of Geophysics, China Earthquake Administration, Beijing 100081, China \\ ${ }^{2}$ Institute of Geology and Geophysics, Chinese Academy of Sciences, Beijing 100029, China \\ ${ }^{3}$ A F Ioffe Physical-Technical Institute, Russian Academy of Sciences, St. Petersburg 194021, Russia
}

\begin{abstract}
The experimental results of 3-D fracture under compression are introduced in brief and the theory of stress criterion of 3-D fracture is studied. Methods to imitate initial fractures are developed. It is pointed that there are important defects in the extreme value (EV) method ever proposed by Palaniswamy and Knauss. The major defect lies in that only two Euler angles (2EA) are considered, but another one is neglected. If the variation of all the three Euler angles (3EA) are considered, one can get better result which is consistent with the observation of faulting that extends on curved surfaces but not on planes. The method of evaluating maximal normal stress direction vector (NSDV) is proposed and further proved to be equivalent to the $3 \mathrm{EA}$ method. It is proved that the NSDV method can be further optimized to the method of composition of the first principal differential plane (CFPDP). The results from CFPDP method can fit the curved surfaces of initial growth observed in the experiments of 3-D fracture. The CFPDP method can also be used to interpret the 3-D fractures of the slipping section between the asperities in the buried fault plane that is modeled as ellipse crack. The results of 3-D fracture can be applied to interpreting the related problems of faulting including the mechanism of a lot of shatter rocks with different dimensions, the cause of earthquakes occurred at the edge of plate under low shear stress, and the mechanism of anisotropy caused by the extensive dilatancy anisotropy (EDA) cracks.
\end{abstract}

Key words: fracture mechanics; 3-D fracture; fault; fracture criterion

CLC number: P315.8 Document code: A

\section{Introduction}

During the conduction of the Wenchuan Earthquake Fault Scientific Drilling (WFSD) project, Fan et al. (2009) found a great deal of break rocks in the in-situ fault drilling WFSD-1 in Wenchuan, of which part was fractured in the Wenchuan earthquake, and part has pre-existed. Tian et al. (2009) studied the seismic velocity anomalies of Longmenshan fault zone after Wenchuan $M_{\mathrm{S}} 8.0$ earthquake and proposed that it is caused by the rock break of the fault during the aftershocks of Wenchuan $M_{\mathrm{S}} 8.0$ earthquake. More and more

\footnotetext{
* Received 10 May 2011; accepted in revised form 24 May 2011; published 10 June 2011.

^ Corresponding author. e-mail: geolisy@cea-igp.ac.cn

(c) The Seismological Society of China and Springer-Verlag Berlin Heidelberg 2011
}

evidences show that the earthquake faulting is not along plane without width, but a break interlayer with definite width. According to Ding et al. (2004), in the rupture process of Kunlun earthquake $\left(M_{\mathrm{S}} 8.1\right)$ on November 14, 2001 , the rupture fault in deep is much wider than that on ground surface. The width of rupture on ground surface along a measuring line is only $2 \mathrm{~m}$, but it approaches to $100 \mathrm{~m}$ in the depth of $30 \mathrm{~m}$. As well known, it is found that there is a lot of break rock and fault gouge in the fault zone. The fault gouge forms mylonite under high temperature and high pressure.

The above phenomena show that the earthquake faulting is quite complicated and it can not be interpreted simply as 2-D shear slipping of fault plane, but a 3 -D fracture. The 3-D fracture means natural fracture of 3-D crack (or fault), e.g., the surface or interior crack (fault) are often modeled as semi-ellipse or ellipse crack. 


\subsection{Several experiments of 3-D fracture}

The earliest experiment of 3-D fracture is Hertz contact that is formed by compressing a cylindrical steel indenter of the diameter, $1.59 \mathrm{~mm}, 3.13 \mathrm{~mm}$ and 6.35 $\mathrm{mm}$, respectively, on the block of Silicate glass (Roesler, 1956). Geometry and loading are shown in Figure 1a. Conical crack extends from the edge of contact area, the angle between the generatrix of the cone and the symmetry axis is about $68.5^{\circ}$ (Figure $1 \mathrm{~b}$ ).

The geometry and loading for mode I and mode III fracture (Sommer, 1969) are shown in Figure 2a. A crack is formed on a circular specimen made up of AR- glass by using a razor blade. The torsion and tensile forces are applied to the specimen at the same time. The tensile loading is acted by the high pressure water filling into the gap of the crack. One can observe double lances-shape initial growth (Figure 8b) and a lot of fracture extending to the outer edge of the specimen (Figure 2b) by using electron microscope.

The geometry and loading for mode III (anti-planeshear) fracture (Knauss, 1970) are shown in Figure 3a. The material of specimen is Solithane 113 glass composed of a two-component cross-linked polymer in the $50 / 50$ composition. The crack was produced with a
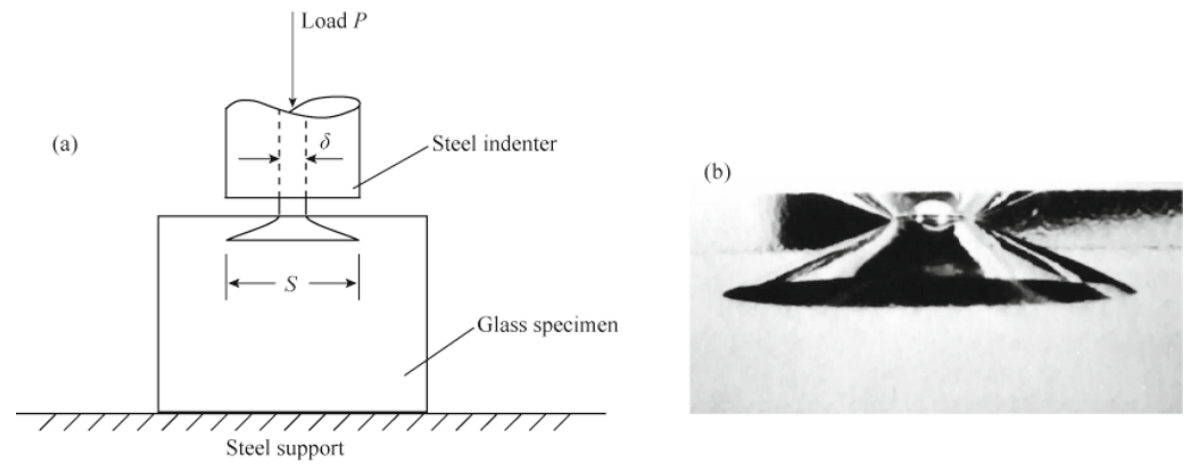

Figure 1 Cone-shaped fracture surface at the edge of contact area of glass specimen (Roesler, 1956). (a) Geometry; (b) View of crack extension.
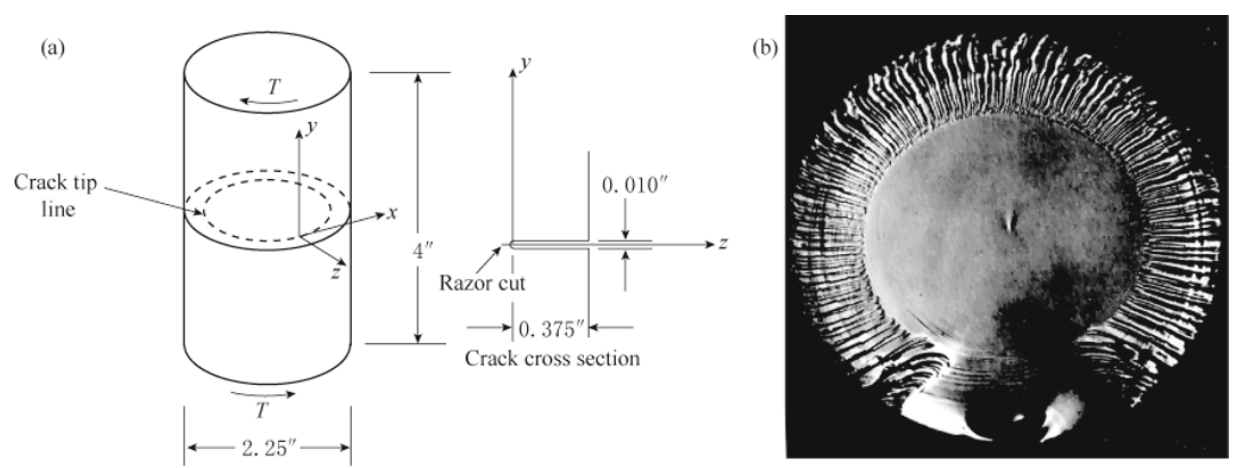

Figure 2 Modes I and III fracture (Sommer, 1969). (a) Geometry where $1^{\prime \prime}=1$ inch (25.40 mm); (b) Extending of crack.

$0.254 \mathrm{~mm}$ thick hollow ground jeweler saw blade and the bottom of the crack was extended $0.254 \mathrm{~mm}$ with a razor blade. Brass flat stock was glued to the flanks of the crack for applying the shear force to the specimen in an instron tester. These bars were kept parallel to each other during the loading. Multiple para-helicalshaped fractures appear at the front of cracks, as shown in Figure 3b.

\subsection{Normal stress fracture criterion}

Figures $2 \mathrm{~b}$ and $3 \mathrm{~b}$ show that the 3 -D crack does not extend along its own plane but generate multiple twisted and turned tensile fracture surfaces. There are two intersecting lines between the fracture surface and the original crack plane. As well known, there is only one intersecting line between two planes. In such way, it is obvious that these initial fractures are not planes 


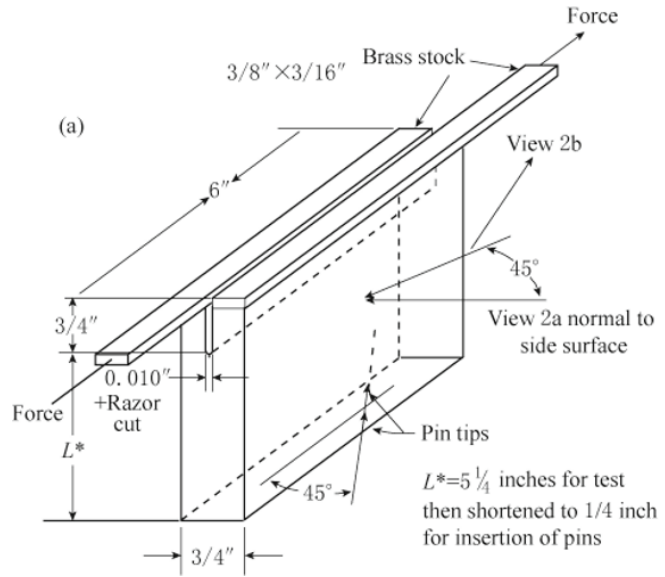

Figure 3 Pure mode III (anti-shear) fracture (Knauss, 1970). (a) Geometry; (b) Close-up view of crack extension.

but curved surfaces.

Summarizing these 3-D fracture experimental results, Palaniswamy and Knauss (1978) proposed the normal stress criterion: (1) The fracture extends along the direction perpendicular to the maximal principle stress $\sigma_{1} ;(2)$ The initial fracture starts when $\sigma_{1}$ near the front of crack approaches to critical value.

\subsection{Imitation of the initial fracture surfaces}

Palaniswamy and Knauss (1978) proposed a method to imitate the 3-D fracture surfaces by using double Euler angles (2EA) (Figure 4). Suppose the orthogonal coordinates of the point $P$ is $(x, y, z)$, and its cylinder coordinate is $(r, \theta, z)(-\pi \leq \theta \leq+\pi) . z$-axis is the tangent line of the crack front, and $x$ - $z$-plane coincides with the original crack plane (Figure 4). They computed the maximum of normal stress $\sigma_{\mathrm{N}}$ in the plane obtained by rotating the $x-z$ plane about the $z$-axis (locally tangent to the crack front) by an angle $\delta$ and then

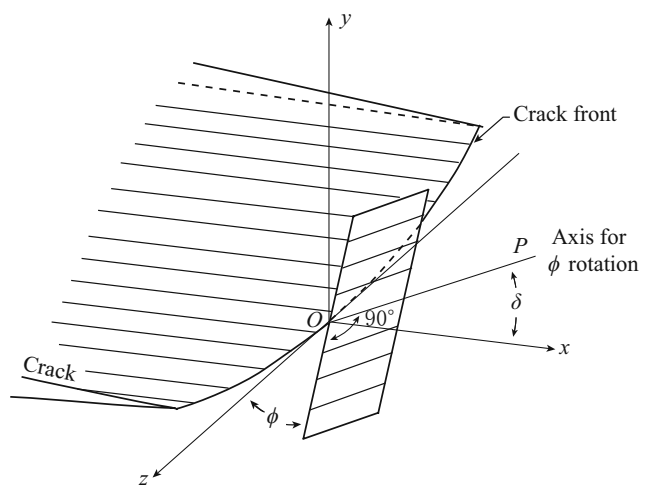

Figure 4 Local coordinate system at the front of a crack and definition of orientation of crack extension plane (Palaniswamy and Knauss, 1978). rotating about the new $x$-axis (say, $x^{\prime}$ axis) by an angle $\phi$ to get the resulting plane (Figure 4). However, they did not give the results of imitation. In fact, the imitating result by using the $2 \mathrm{EA}$ method is only a plane but not a curved surface.

Li (1990,1991) proposed a method of composition of the first principal differential planes (CFPDP) and successfully imitated the 3-D initial curved surface. But he did not point out the defect of the 2EA method presented by Palaniswamy and Knauss (1978), and did not illustrate the difference of CFPDP method from the $2 \mathrm{EA}$ method. In such case, the theory of 3-D fracture needs to be further improved.

\section{Experiments of 3-D crack prop- agation under compression}

As most of the faults are under compression and belong to fracture of mode II and III, it is necessary to introduce the experimental results and to have a comprehension of mode II and mode III fracture (Teng et al., 1987, Yin et al., 1989, 1991).

\subsection{Geometry and loading description}

The non-penetrated (surface of semi-ellipse) crack is pre-generated in the center of a plate and is oblique to the direction of uniaxial compression loading (Yin et al., 1988; Li, 1991). The crack surfaces are under shear loading and are closed in glass and organic glass when the specimen is pressed. No lubrication oil is filled into the crack surfaces in group $A$ of silicate glass specimen, but in group $B$ of silicate glass specimen, some engine oil is filled into the crack surfaces. 


\subsection{Geometry and principle of the experiment}

An orthogonal coordinate system is set up, as shown in Figure 5. Suppose the $x$-axis is along the major axis of the semi-ellipse, the $y$-axis is along the minor axis of the semi-ellipse and both are perpendicular to the surface of the plate. The $z$-axis is perpendicular to

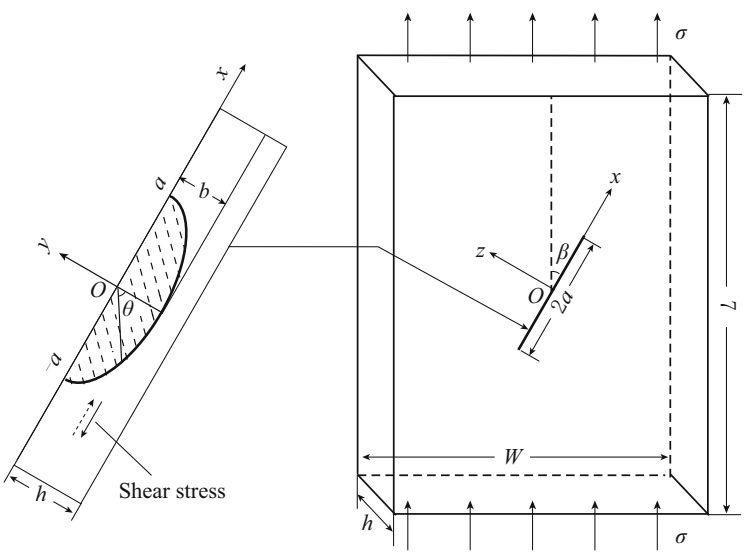

Figure 5 Geometry of 3-D fracture of surface crack under uni-axial press. The left is the local side looking of crack. $h$ is the thickness of the plate of the specimen, $a$ is the length of half major axis and $b$ is the length of half minor axis of the ellipse.

the crack surface. The origin point $O$ is located in the centers of the semi-ellipse. $x$ -,$y$ - and $z$-axis consists of a right hand coordinate system (Figure 5). The angle $\beta$ between the direction of the semi-ellipse and the press loading $\sigma$ is called crack angle. For uniaxial loading, the boundary conditions are

$$
\begin{gathered}
\sigma_{x x}=-\sigma \cos ^{2} \beta, \quad \sigma_{z z}=-\sigma \sin ^{2} \beta, \\
\tau_{x z}=-\sigma \sin \beta \cos \beta,
\end{gathered}
$$

where $\sigma$ is positive for tensile. For simplicity, the friction along the crack surfaces is supposed as uniform. So the valid shear stress along the crack surface is

$$
\tau_{\mathrm{e}}=\tau_{x z}-f \sigma_{z z},
$$

where $f$ is frictional coefficient. The stress intensity factors are (China Academy of Aeronautics, 1981, 1993)

$$
K_{\mathrm{II}}=M_{2} \tau_{\mathrm{e}} \frac{\sqrt{\pi b}}{E(k)}, \quad K_{\mathrm{III}}=M_{3} \tau_{\mathrm{e}} \frac{\sqrt{\pi b}}{E(k)},
$$

where $E(k)$ is second kind of complete ellipse integral,

$$
\begin{gathered}
E(k)=\int_{0}^{\pi / 2} \sqrt{1-k^{2} \sin ^{2} \varphi} \mathrm{d} \varphi, \\
k^{2}=1-\frac{b^{2}}{a^{2}} .
\end{gathered}
$$

$M_{2}$ and $M_{3}$ can be consulted in Figure 6. From the above principle, one can know that mode III fracture may be generated at $\Omega=0$ and $M_{2}=0$, mode II fracture may be generated at $\Omega= \pm 90^{\circ}$ and $M_{3}=0$, and fracture of modes II and III with different ratios of $K_{\text {II }} / K_{\text {III }}$ may be generated at the other $\Omega$, where $K_{\mathrm{II}} / K_{\mathrm{III}}=M_{2} / M_{3}$.

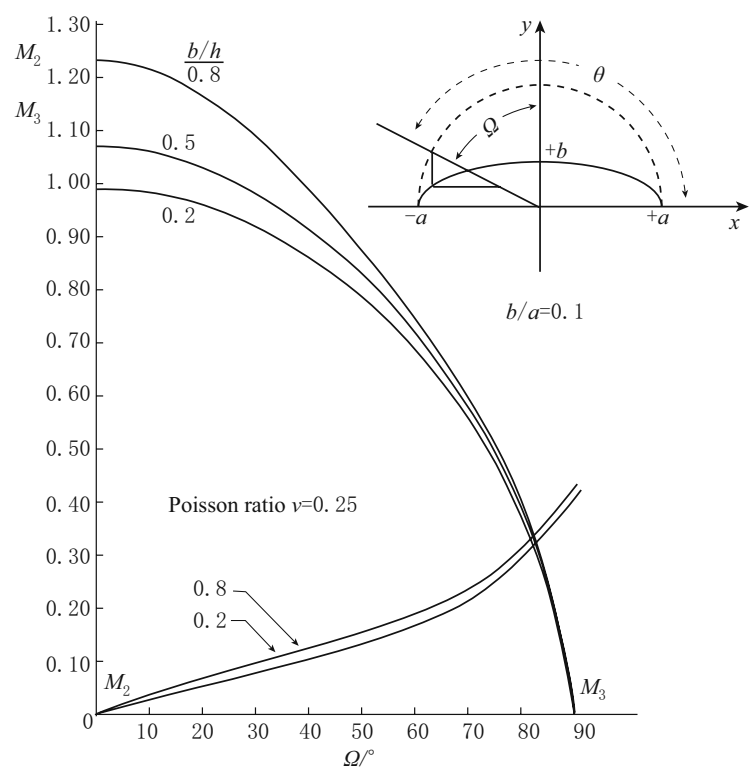

Figure $6 \quad M_{2}$ and $M_{3}$ values at the crack front when the crack in the surface of a plate that is under uniform shear loading (China Academy of Aeronautics, 1981, 1993).

The geometry of our experiment is referred to China Academy of Aeronautics $(1981,1993)$. In this handbook, the geometry of a crack in the surface of a plate is listed into the section named " 3 -D crack", including $b / h=0.2,0.6,0.8, b / a=0.1,0.2$. In our experiment, the cases including $h=5 \mathrm{~mm}, b / h \approx 0.6, b / a \approx 0.15$ are all contained in the handbook. In such way, there is no doubt that the geometry of our experiment belongs to 3 -D crack.

\subsection{Observation of experiments in glass speci- men}

When the loading approaches to critical value, the front of original crack start to grow, one can observe the following phenomena.

1) The first extension appears in the middle of the crack (edge of short axis), then the extensions spread to the tips, at last extensions appear at the tips of major axis.

2) Hundreds of discrete initial fractures appear at the length of $25 \mathrm{~cm}$, as shown in Figure $7 \mathrm{a}$. The initial extensions merge into each other in the process of 


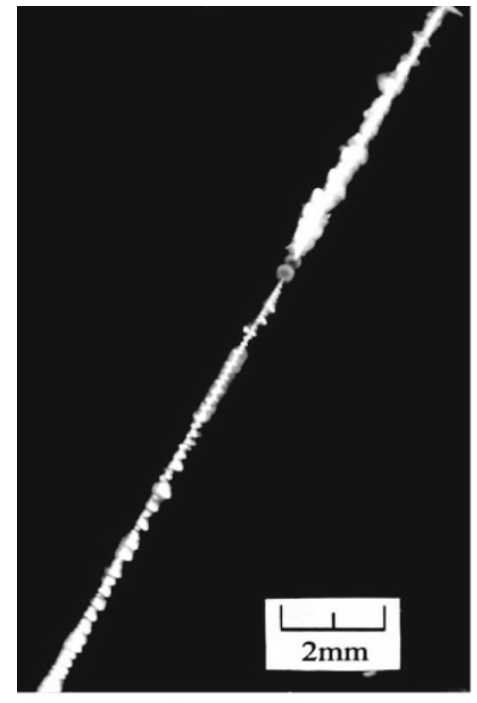

(a)

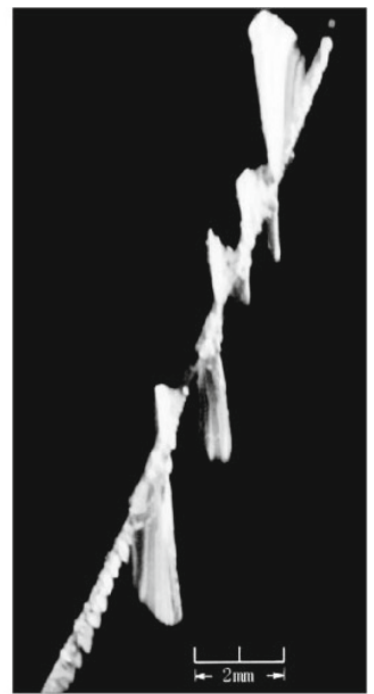

(b)
Figure 7 3-D initial extensions from the front of surface crack in glass specimen.

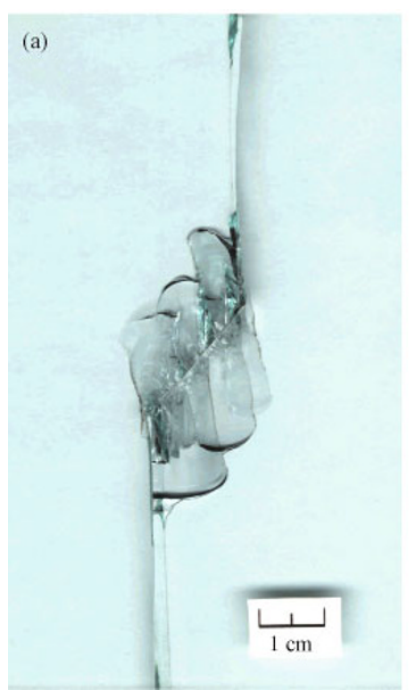

propagation, its number decreases gradually, but the distance keeps equal. The value of distance equals roughly the radius of the fracture surface, as shown in Figure $7 b$.

3) The shapes of the fracture surfaces are like parahelical, but the sizes are different. Where the extending in the middle of the original crack is the shortest, the extending at the tips of major axes are the longest.

4) The shape of every crack extension is referred to parahelical but one side is longer than another. These extensions array directionally and appear as en echelon or saw-tooth in the surface of plate as shown in Figures $7 \mathrm{~b}$ and 8 . In Figure $8 \mathrm{a}$, the crack with $\beta \approx 39^{\circ}$ in group $A$ breaks out and extends like bean valve caused by stick-slip between the surfaces of crack. In Figures $8 \mathrm{~b}-$ $8 \mathrm{~d}$, the crack of group $B$ grows steadily with the loading increasing slowly and uniformly. In Figure $8 \mathrm{~d}, \beta \approx 69.2^{\circ}$, the location of main fracture surface is not at the tip of the major axis but near the middle section.
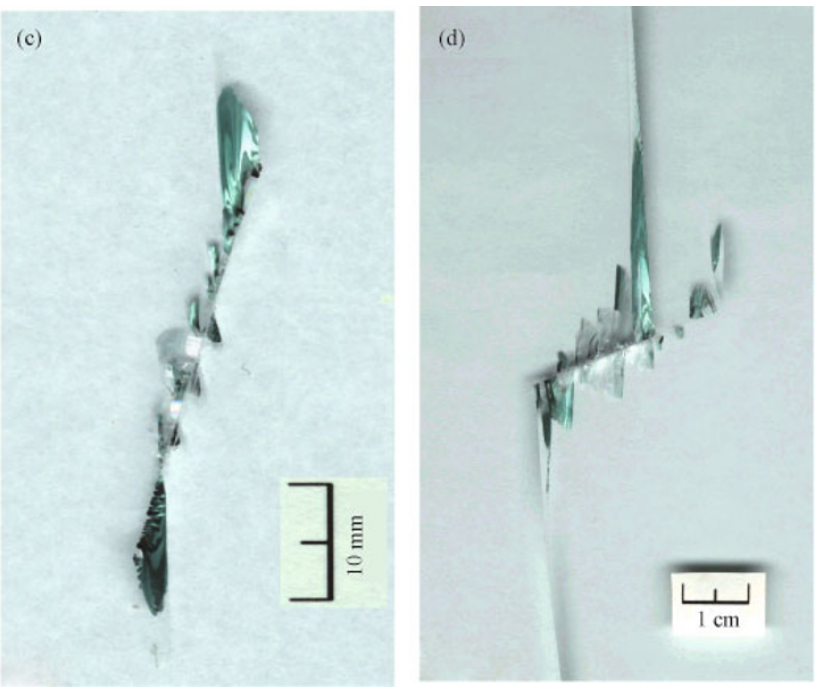

Figure 8 Extensions generated from the front of surface crack in glass specimen. (a) Group $A, \beta \approx 39^{\circ}$; (b) Group $B, \beta \approx 45^{\circ} ;$ (c) Group $B, \beta \approx 15^{\circ} ;\left(\right.$ d) Group $B, \beta \approx 69.2^{\circ}$.

It seems that it is related to the asperity in the crack surfaces.

In Figure 8b, one can see many spoke shaped stripes that start from the initial points similar to that in Figure 3b. Lawn and Wilshaw (1975) thought that it is a kind of branch phenomenon and discussed its mechanism. In the extension surfaces in Figure 8, one can see many interfered stripes of light, which can be seen as the results of interference of the incident light and the reflect light between the micro-opened crack surfaces.

\subsection{Analysis of experimental results}

\subsubsection{Order of extension initial}

From equation (3), we have $K_{\mathrm{II}} / K_{\mathrm{III}}=M_{2} / M_{3}$. In Figure 6 , it can be seen that $\left.\left.M_{2}\right|_{\Omega= \pm 90^{\circ}} \ll M_{3}\right|_{\Omega=0^{\circ}}$, thus $\left.\left.K_{\mathrm{II}}\right|_{\Omega= \pm 90^{\circ}} \ll K_{\mathrm{III}}\right|_{\Omega=0^{\circ}}$. In such way, in different points at the crack edge, the critical loading values are different. At the point $\Omega=0$, the critical condition is 
approached firstly, and the mode III fracture is initiated. Then at $0^{\circ}<|\Omega|<90^{\circ}$, the fractures with different ratios of $K_{\text {II }} / K_{\text {III }}$ are initiated one by one. At the point $\Omega= \pm 90^{\circ}$, the critical condition is approached at last, and the mode II fracture is initiated. Here the extensions are the longest and are called main fracture.

\subsubsection{Condition of fracture initiation}

Substituting equations (1) and (2) into equation (3), we have

$$
\begin{aligned}
K_{J}= & h_{j}[\sin 2 \beta \cos \beta-f(1-\cos 2 \beta)] \\
& (J=\mathrm{II}, \mathrm{III} ; j=1,2),
\end{aligned}
$$

where $h_{j}=-\left[M_{j} / 2 E(k)\right] \sigma \sqrt{\pi b}$. In critical condition, $\left|K_{J}\right|=K_{J \mathrm{c}}, \sigma=\sigma_{\mathrm{c}}$, and then $h_{j}=h_{\mathrm{c}}$, substituting the above-mentioned into equation (5), we have

$$
\left|h_{\mathrm{c}}\right|=\frac{K_{J \mathrm{c}}}{\sin 2 \beta-f(1-\cos 2 \beta)} .
$$

In equation (6), only $\beta$ is variable. It can be proved that there is a minimum of $h_{\mathrm{c}}$ at $\beta=\beta_{\mathrm{m}}$, as shown in Figure 9 , denoted as $h_{\mathrm{cm}}$. That means the fracture initiates easiest when $\beta=\beta_{\mathrm{m}}$.

$$
\tan 2 \beta_{\mathrm{m}}=\frac{1}{f} \quad \text { or } \quad \beta_{\mathrm{m}}=\frac{1}{2} \arctan \frac{1}{f}
$$

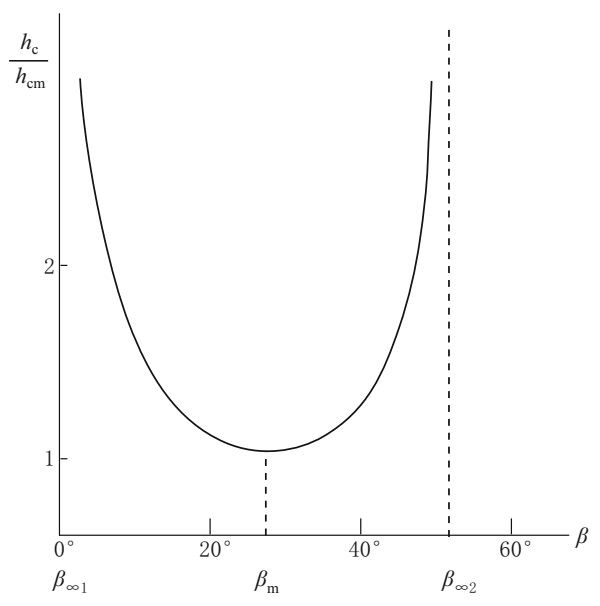

Figure 9 Graph of $h_{\mathrm{c}}$ as a function of $\beta$.

The relationship between $f$ and $\beta_{\mathrm{m}}$ are shown in Table 1 . Besides, there are two points at $\beta_{\infty 1}$ and $\beta_{\infty 2}$ in which $h_{\mathrm{c}}$ is infinite.

$$
\beta_{\infty 1}=0, \quad \beta_{\infty 2}=\arctan \frac{1}{f} .
$$

That means $h_{\mathrm{c}}$ becomes infinite when $\beta \rightarrow \beta_{\infty 1}$ (decreases to approach) or $\beta \rightarrow \beta_{\infty 2}$ (increases to approach), as shown in Figure 9. It is very difficult to make the crack start to extend. Comparing equation (8) with equation (7), one can see that $\beta_{\infty 2}=2 \beta_{\mathrm{m}}$ (Figure 9).

Table 1 Relationship between $f$ and $\beta_{\mathrm{m}}$

\begin{tabular}{ccccccc}
\hline$f$ & 0.8 & 0.7 & 0.6 & 0.5 & 0.4 & 0.3 \\
\hline$\beta_{\mathrm{m}}$ & $25^{\circ} 40^{\prime}$ & $27^{\circ} 30^{\prime}$ & $29^{\circ} 31^{\prime}$ & $31^{\circ} 43^{\prime}$ & $34^{\circ} 06^{\prime}$ & $36^{\circ} 39^{\prime}$ \\
\hline
\end{tabular}

Through experiment, Yin et al. (1988) obtained $f \approx 0.76$ for group $A$ and $f \approx 0.56$ for group $B$ between the surfaces of crack in silicate glass. According to Mohr criterion, the pore pressure of lubrication oil offsets part of normal stress acting on the crack surface. If the pore pressure is not included in, the friction coefficient in group $B$ is low. From Table 1 , we can see that $\beta_{\infty 2} \approx 52^{\circ}$ for group $A$, and $\beta_{\infty 2} \approx 60^{\circ}$ for group $B$. Obviously, for the specimen showed in Figures $8 \mathrm{a}-8 \mathrm{c}, \beta$ is smaller than $\beta_{\infty 2}$, whereas for the specimen in Figure $8 \mathrm{~d}, \beta\left(\approx 69.2^{\circ}\right)$ is much larger than $\beta_{\infty 2} \approx 60^{\circ}$. The direction of maximal principle stress is near perpendicular to the crack surface. The shear stress is very low, but the extensions still generate, except that the location of main fracture is stochastical instead of at the tip of the origin crack. From Table 1 , in this places, if only $f<0.35, \beta_{\infty 2}$ can be near $70^{\circ}$ and this part becomes sliding section. Despite of the crack angle is very large, and then the shear stress is very low, the static friction force can still be overcome, and therefore the main fractures appear at the tip of the sliding section.

The experimental phenomenon shown in Figure 8d can be used to interpret similar case of earthquake fault at plate margin. An interesting fact is that for fault located at the plate margin the shear stress is very low, even is much lower than that of intraplate faults. The results of stress measurements in the drilling hole (depth of $304.8 \mathrm{~m}$ ) at Cajon Pass near the San Andreas fault and many earthquake source mechanisms show that the direction of maximal principal stress is almost perpendicular to the fault. This result shows that the shear stress in the fault is very low (Ruff, 2002). In recent years, a common recognition has been obtained that the earthquakes with low stress occurred at the margin of plate should be resulted from effect of pore pressure of water from a lot of observation data especially from the deep drilling hole (Ruff, 2002).

\subsection{Experimental research of acoustic emission} (AE) of 3-D fracture in rock specimen

In 1999, Institute of Geophysics, China Earthquake Administration, cooperated with A F Ioffe PhysicalTechnical Institute, Russian Academy of Science, stud- 
ied experimentally decimeter scale rock fracture. The SDAE-8 AE real time sampling and locating system (provided by Pro. Kuksenko) and Changjiang-500 testing machine are used (Li et al., 2000, 2009).

Figures 10a-10c show the location of AE events during the process of extension of the non-penetrated notch of marble. where $2 a$ is the length of the maior axis of semi-ellipse. Most of the AE events centralize along the arc-like line of the notch front. These AE events disperse gradually as the loading increase slowly, indicating that multiple extensions appear on the points in arc-like line front of the notch. As contrast, the AE events always centralize at the two tips of the penetrated notch of the rock specimen. as shown in Figures 10d-10f.
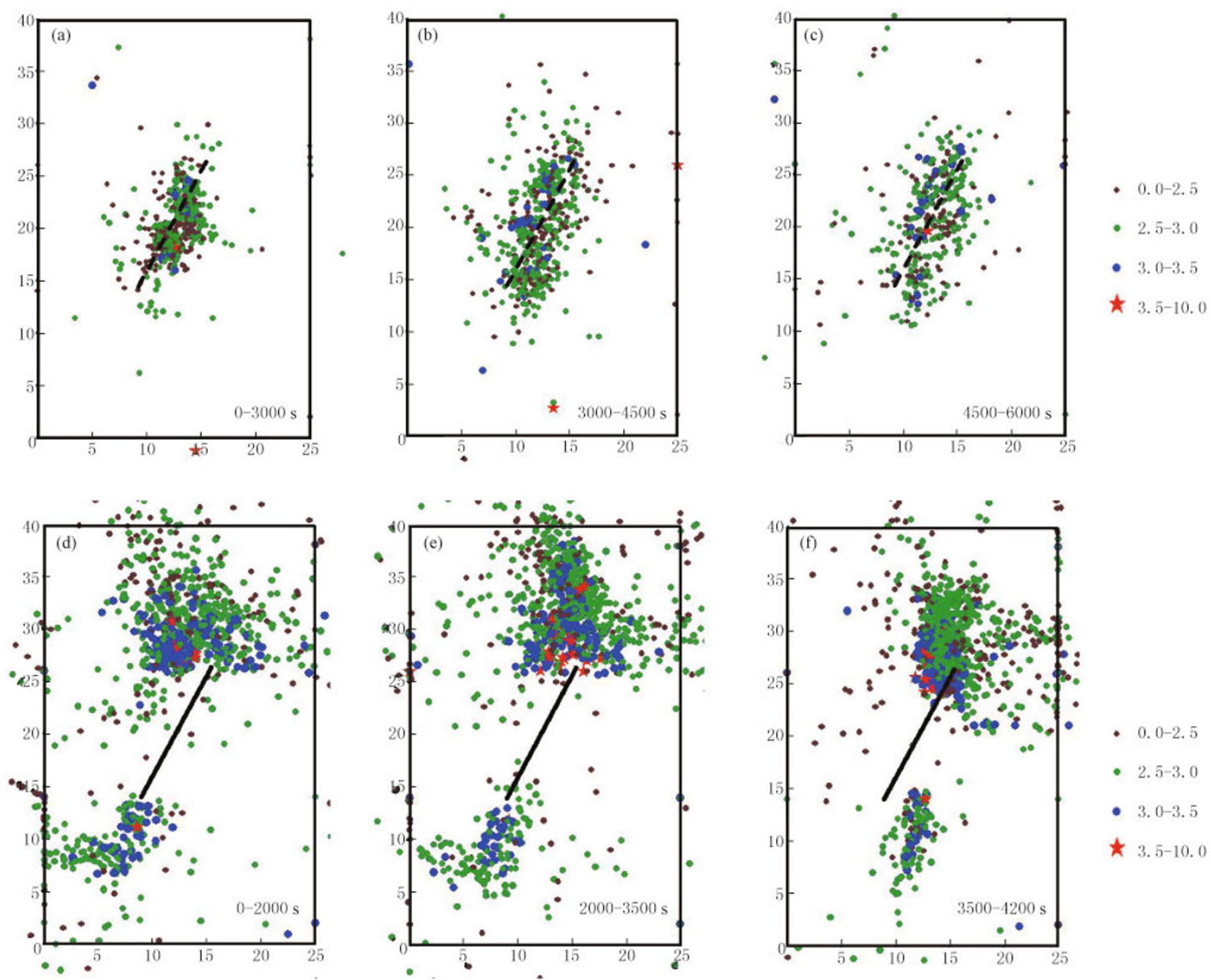

Figure $10(\mathrm{a}-\mathrm{c})$ Location of $\mathrm{AE}$ during the process of propagation of non-penetrated notch in a plate of marble No. 991018 with size $400 \mathrm{~mm} \times 250 \mathrm{~mm} \times 41 \mathrm{~mm}$, length of the major axis $2 a=172 \mathrm{~mm}$ and crack angle $\beta=30^{\circ}$. (d-f) Contrast of penetrated notch in another plate of marble No. 991015 with size $399 \mathrm{~mm} \times 248$ $\mathrm{mm} \times 39 \mathrm{~mm}$, length of the notch $2 a=143 \mathrm{~mm}$ and $\beta=29^{\circ}$.

\section{Theoretical research of $3-\mathrm{D}$ ini-} tial fracture

\subsection{Method of evaluating $\sigma_{\mathrm{Nmax}}$ by using three Euler angles (3EA)}

There are two reasons for that the $2 \mathrm{EA}$ method is not quite successful. Firstly, the size of differential plane is enlarged arbitrarily; secondly, only two Euler angles of $\delta$ and $\phi$ are considered while the variations of $\psi$-angle is neglected (Figure 11). In fact, $\psi=0$ is always default in their method. In this way, only one plane but not curved surface solution is obtained.

We set up the coordinate just as the same as Figure 4. According to Lawn and Wilshaw (1975), the components of stress near the front of crack are 


$$
\left\{\begin{array}{l}
\sigma_{x x}=\frac{K_{\mathrm{I}}}{\sqrt{2 \pi r}} \cos \frac{\theta}{2}\left(1-\sin \frac{\theta}{2} \sin \frac{3}{2} \theta\right)-\frac{K_{\mathrm{II}}}{\sqrt{2 \pi r}} \sin \frac{\theta}{2}\left(2+\cos \frac{\theta}{2} \cos \frac{3}{2} \theta\right)+o\left(r^{-1 / 2}\right) \\
\sigma_{y y}=\frac{K_{\mathrm{I}}}{\sqrt{2 \pi r}} \cos \frac{\theta}{2}\left(1+\sin \frac{\theta}{2} \sin \frac{3}{2} \theta\right)+\frac{K_{\mathrm{II}}}{\sqrt{2 \pi r}} \sin \frac{\theta}{2} \cdot \cos \frac{\theta}{2} \cdot \cos \frac{3}{2} \theta+o\left(r^{-1 / 2}\right) \\
\tau_{x y}=\frac{K_{\mathrm{I}}}{\sqrt{2 \pi r}} \sin \frac{\theta}{2} \cdot \cos \frac{\theta}{2} \cdot \cos \frac{3}{2} \theta+\frac{K_{\mathrm{II}}}{\sqrt{2 \pi r}} \cos \frac{\theta}{2}\left(1-\sin \frac{\theta}{2} \sin \frac{3}{2} \theta\right)+o\left(r^{-1 / 2}\right) \\
\tau_{x z}=-\frac{K_{\mathrm{III}}}{\sqrt{2 \pi r}} \sin \frac{\theta}{2}+o\left(r^{-1 / 2}\right), \tau_{y z}=\frac{K_{\mathrm{III}}}{\sqrt{2 \pi r}} \cos \frac{\theta}{2}+o\left(r^{-1 / 2}\right) \\
\sigma_{z z}= \begin{cases}0 \quad \text { for } \operatorname{plane} \operatorname{stress} & \text { for plane strain } \\
2 \nu\left(\frac{K_{\mathrm{I}}}{\sqrt{2 \pi r}} \cos \frac{\theta}{2}-\frac{K_{\mathrm{II}}}{\sqrt{2 \pi r}} \sin \frac{\theta}{2}\right)+o\left(r^{-1 / 2}\right)\end{cases}
\end{array}\right.
$$

where $K_{\mathrm{I}}, K_{\mathrm{II}}, K_{\mathrm{III}}$ are the stress intensity factors, $\nu$ is Poisson ratio.

Next we will discuss the $3 \mathrm{EA}$ method. This paper takes $0 \leq \psi \leq 2 \pi$ (Figure 11) in general situations. In order to search for the maximum of $\sigma_{\mathrm{N}}$, we need to rotate $x$-z-plane about $O$-point by using three Euler angles $\delta$, $\phi$ and $\psi(3 \mathrm{EA})$ as the following steps.

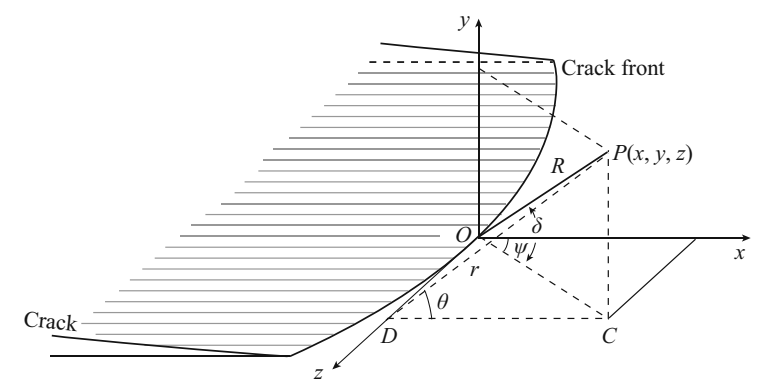

Figure 11 Coordinate of the crack front.

1) Rotating the $x y z$ coordinate system about $y$ axis by an angle $\psi$, the $x$ - $z$-plane turns to be $x_{1}-z_{1}$-plane in $x_{1} y_{1} z_{1}$ coordinate system.

2) Rotating the $x_{1} y_{1} z_{1}$ coordinate system about $z_{1}$-axis by an angle $\delta$, the $x_{1}-z_{1}$-plane turns to be $x_{2}$ $z_{2}$-plane in $x_{2} y_{2} z_{2}$ coordinate system.

3) Rotating the $x_{2} y_{2} z_{2}$ coordinate system about the $x_{2}$-axis by an angle $\phi$, the $x_{2}-z_{2}$-plane turns to be $x_{3}$ $z_{3}$-plane in $x_{3} y_{3} z_{3}$ coordinate system. Then the stress tensor in $x_{3} y_{3} z_{3}$ coordinate is

$$
\boldsymbol{\sigma}_{i_{3} j_{3}}=\boldsymbol{\alpha}_{23}\left[\boldsymbol{\alpha}_{12}\left(\boldsymbol{\alpha}_{01} \boldsymbol{\sigma}_{i j} \boldsymbol{\alpha}_{01}^{\mathrm{T}}\right) \boldsymbol{\alpha}_{12}^{\mathrm{T}}\right] \boldsymbol{\alpha}_{23}^{\mathrm{T}} .
$$

where

$$
\boldsymbol{\sigma}_{i j}=\left(\begin{array}{ccc}
\sigma_{x x} & \tau_{x y} & \tau_{x z} \\
\tau_{x y} & \sigma_{y y} & \tau_{y z} \\
\tau_{x z} & \tau_{y z} & \sigma_{z z}
\end{array}\right)
$$

$$
\begin{aligned}
& \boldsymbol{\alpha}_{23}=\left(\begin{array}{ccc}
1 & 0 & 0 \\
0 & \cos \phi & \sin \phi \\
0 & -\sin \phi & \cos \phi
\end{array}\right), \\
& \boldsymbol{\alpha}_{12}=\left(\begin{array}{ccc}
\cos \delta & \sin \delta & 0 \\
-\sin \delta & \cos \delta & 0 \\
0 & 0 & 1
\end{array}\right), \\
& \boldsymbol{\alpha}_{01}=\left(\begin{array}{ccc}
\cos \psi & 0 & \sin \psi \\
0 & 1 & 0 \\
-\sin \psi & 0 & \cos \psi
\end{array}\right),
\end{aligned}
$$

then

$$
\sigma_{\mathrm{N}}=\sigma_{y_{3} y_{3}}
$$

Based on the specified value of $\psi, \sigma_{\mathrm{Nmax}}$ can be deduced from the solution of the following equations:

$$
\frac{\partial \sigma_{\mathrm{N}}}{\partial \delta}=0, \quad \frac{\partial \sigma_{\mathrm{N}}}{\partial \phi}=0
$$

Suppose $\delta_{0}$ and $\phi_{0}$ are roots of the above equations. Substituting $\delta_{0}$ and $\phi_{0}$ into the left side of the following formulae, one can verify whether the following inequalities are satisfied.

$$
\begin{gathered}
\left.\frac{\partial^{2} \sigma_{\mathrm{N}}}{\partial \delta^{2}}\right|_{\substack{\phi=\phi_{0} \\
\delta=\delta_{0}}}<0,\left.\quad \frac{\partial^{2} \sigma_{\mathrm{N}}}{\partial \phi^{2}}\right|_{\substack{\phi=\phi_{0} \\
\delta=\delta_{0}}}<0, \\
{\left.\left[\frac{\partial^{2} \sigma_{\mathrm{N}}}{\partial \delta^{2}} \frac{\partial^{2} \sigma_{\mathrm{N}}}{\partial \phi^{2}}-\left(\frac{\partial^{2} \sigma_{\mathrm{N}}}{\partial \delta \partial \phi}\right)^{2}\right]\right|_{\substack{\phi=\phi_{0} \\
\delta=\delta_{0}}}>0 .}
\end{gathered}
$$

Now, the normal direction of $x_{3}-z_{3}$-plane is

$$
\boldsymbol{n}_{s}=\boldsymbol{O} \boldsymbol{y}_{3}(x, y, z)=(l, m, n)=
$$

$(-\cos \phi \sin \delta \cos \psi-\sin \phi \sin \psi, \cos \varphi \cos \delta$,

$$
-\cos \phi \sin \delta \sin \psi+\sin \phi \cos \psi) \text {. }
$$

From the geometry in Figure 11, one can get

$$
\tan \theta=\sec \psi \cdot \tan \delta, \quad r=\frac{R \sin \delta}{\sin \theta} .
$$


From the above equations, we can draw that if $\psi \neq 0$, then $\theta \neq \delta$, whereas, only if $\psi=0$, then $\theta=\delta$.

Let $\psi=0$ in equation (14), then $\delta=\theta$, the conditions are reduced to the situation treated by Yin et al. (1991). It can be seen that the 3EA method proposed by Palaniswamy and Knauss (1978) can only deduce a special differential plane with $\psi=0$ that is called zero line.

\subsection{Method of evaluating maximal normal stress with directional vector (NSDV) \\ 3.2.1 Maximal normal stress}

The first step of the normal stress with directional (NSDV) method is to rotate the differential plane $M$ at the point $P\left(r, \theta_{0}, z\right)$ near the initial point to find a normal direction $\boldsymbol{n}=(l, m, n)$, in which the normal stress $\sigma_{\mathrm{N}}$ is maximum. The differential plane is denoted as $M_{\max }$, its normal direction is $\boldsymbol{n}_{\max }\left(l_{0}, m_{0}, n_{0}\right)$.

According to the Cauchy equation,

$$
\begin{array}{r}
\sigma_{\mathrm{N}}=l^{2} \sigma_{x x}+m^{2} \sigma_{y y}+n^{2} \sigma_{z z}+ \\
2 l m \tau_{x y}+2 m n \tau_{y z}+2 n l \tau_{z x} .
\end{array}
$$

When the conditions

$$
\frac{\partial \sigma_{\mathrm{N}}}{\partial l}=0, \quad \frac{\partial \sigma_{\mathrm{N}}}{\partial m}=0, \quad \frac{\partial \sigma_{\mathrm{N}}}{\partial n}=0
$$

are satisfied, $\sigma_{\mathrm{N}}$ will become $\sigma_{\mathrm{Nmax}}$. With the constraint of the condition $l^{2}+m^{2}+n^{2}=1$, only the 1st and 2nd equations are needed to be solved. Thus, the roots $l_{0}, m_{0}, n_{0}$ are obtained and then substituted into the left of the following formulae so as to verify whether the following inequalities are satisfied.

$$
\begin{gathered}
\left.\frac{\partial^{2} \sigma_{\mathrm{N}}}{\partial l^{2}}\right|_{\substack{l=l_{0} \\
m=m_{0} \\
n=n_{0}}}<0,\left.\quad \frac{\partial^{2} \sigma_{\mathrm{N}}}{\partial m^{2}}\right|_{\substack{l=l_{0} \\
m=m_{0} \\
m=n_{0}}}<0, \\
{\left.\left[\frac{\partial^{2} \sigma_{\mathrm{N}}}{\partial l^{2}} \frac{\partial^{2} \sigma_{\mathrm{N}}}{\partial m^{2}}-\left(\frac{\partial^{2} \sigma_{\mathrm{N}}}{\partial l \partial m}\right)^{2}\right]\right|_{\substack{l=l_{0} \\
m=m_{0} \\
n=n_{0}}}>0 .}
\end{gathered}
$$

\subsubsection{Geometry of constraint condition}

Suppose line $\boldsymbol{O P}$ is one of the initial extensions that are straight segments. Then the direction of $\boldsymbol{O P}$ is

$$
\begin{gathered}
\boldsymbol{O P}=\boldsymbol{R}_{\mathrm{P}}=\left\{x_{\mathrm{P}}-0, y_{\mathrm{P}}-0, z_{\mathrm{P}}-0\right\}= \\
\left\{r \cos \theta_{0}, r \sin \theta_{0}, z_{\mathrm{P}}\left(r, \theta_{0}\right)\right\},
\end{gathered}
$$

where $0 \leq r \leq r_{m},-\pi \leq \theta_{0} \leq \pi$. Naturally $\boldsymbol{R}_{P} \perp$ $\boldsymbol{n}_{\max }\left(l_{0}, m_{0}, n_{0}\right)$, i.e., $\boldsymbol{R}_{P} \cdot \boldsymbol{n}_{\max }=0$, that means

$$
l_{0} r \cos \theta_{0}+m_{0} r \sin \theta_{0}+n_{0} z_{\mathrm{P}}\left(r, \theta_{0}\right)=0 .
$$

That is just the algebraic form of $M_{\max }$. Equation (19) gives the necessary geometry condition that $M_{\max }$ extends through $O$-point. In such way, equation (19) is called geometry constraint equation.

\subsubsection{Construction of initial fracture surface}

The second step of the NSDV method is to construct the initial fracture surface. From equation (19) $z_{P}\left(r, \theta_{0}\right)$ can be obtained, and then is substituted into equation (18), we have

$$
\begin{gathered}
\boldsymbol{R}_{P}=\left\{x_{P}, y_{P}, z_{P}\right\}= \\
r\left\{\cos \theta_{0}, \sin \theta_{0}, \frac{-\left(l_{0} \cos \theta_{0}+m_{0} \sin \theta_{0}\right)}{n_{0}}\right\},
\end{gathered}
$$

and $\boldsymbol{R}_{P}$ is also the expression of $M_{\max }$ that is coincident with $\boldsymbol{O P}$.

Equation (20) holds to $-\pi \leq \theta \leq \pi$. That is to say, equation (20) shows the set of all differential planes extending from the point $O$ in the range of $-\pi \leq \theta_{0} \leq \pi$. It is just this set that constructs the total initial fracture surface extending from the point $O$.

The third step of the NSDV method is to locate the edge of initial fracture surface. We compute the isoline of $\sigma_{1}=\sigma_{m}$ as the edge of initial fracture surface, where $\sigma_{m}$ is anti-tensile strength of the medium.

\subsubsection{Identities of NSDV and 3EA methods}

As mentioned above, the NSDV method and 3EA method can be used independently. From equation (13), we get the relationship between the normal direction vector of $M_{\max }$ after rotating three times and the three Euler angles are

$$
\left\{\begin{array}{l}
l=-\cos \phi \sin \delta \cos \psi-\sin \phi \sin \psi \\
m=\cos \phi \cos \delta \\
n=-\cos \phi \sin \delta \sin \psi+\sin \phi \cos \psi
\end{array} .\right.
$$

From Figure 12, we can deduce $\tan \psi=z_{P} / x_{P}$. By using equations (14), (20) and the 2nd equation of (21), the reverse transformation of equation (21) is

$$
\left\{\begin{array}{l}
\psi=-\arctan \frac{l+m \tan \theta}{n} \\
\delta=\arctan \frac{n \tan \theta}{\sqrt{n^{2}+(l+m \tan \theta)^{2}}} \\
\phi=\arccos \left[m \sqrt{1+\frac{n^{2} \tan ^{2} \theta}{n^{2}+(l+m \tan \theta)^{2}}}\right]
\end{array} .\right.
$$




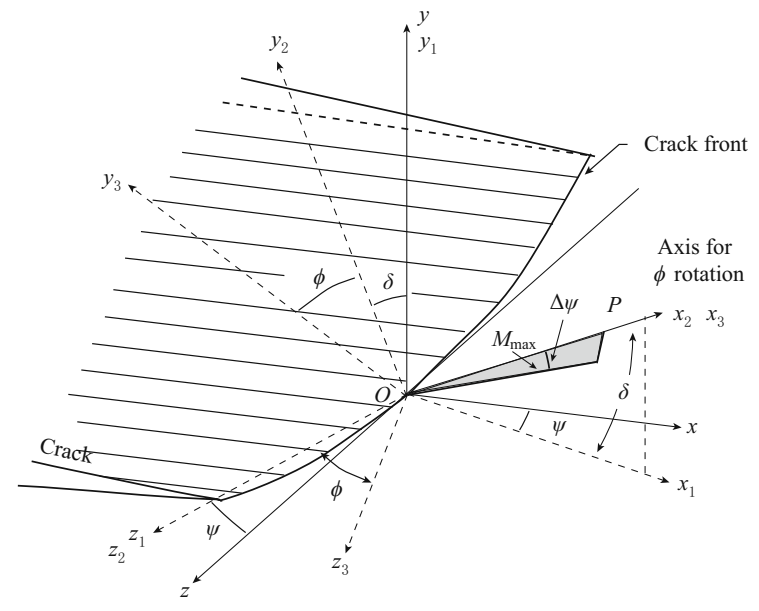

Figure 12 Illustration of three Euler angle method.

Thus it is clear that $3 \mathrm{EA}$ method is equivalent to NSDV method except that the steps are different. In $3 \mathrm{EA}$ method, the geometry constraint condition is restricted at first, then the maximum of $\sigma_{\mathrm{N}}$ is searched. In NSDV method, the orders are just contrary.

The NSDV method is still complicated in calculation, but it is a key step, from which the method of composition of first principal differential planes (CFPDP) can be deduced easily.

\subsection{Method of composition of first principal differential planes (CFPDP)}

\subsubsection{Basis and main points}

The CFPDP method was proposed by Li (1990, 1991) based on the well-known law that the maximal principal stress $\sigma_{1}$ is just the maximal normal stress $\sigma_{\text {Nmax }}$ (Timoshenko and Gooder, 1970; Qian and Ye, 1956) at any point in the continuous medium. So, we only need change from computing the maximal normal stress, which is the first step of NSDV method, to computing the principal stress and the first principal direc- tion at an arbitrary point near the front of the crack and keep the other steps unchanged. That means CFPDP method is identical to the NSDV method. Specially, CFPDP method brings a great simplification in calculation.

The key of CFPDP method lies in whether the following proposition is satisfied: the first principal differential plane $M_{1}$ with normal direction $\boldsymbol{n}_{1}\left(l_{0}, m_{0}, n_{0}\right)$ may intersect with the original crack front (i.e., $z$-axis in Figure 11) in the definition area of $-\pi \leq \theta \leq+\pi$. $\operatorname{Li}(1991)$ and Li et al. (2009) proved that only if $K_{\text {III }} \neq 0$, the geometry constraint condition given by equation (19) can always be satisfied. In such way, the proposition can be tenable under the condition of $K_{\text {III }} \neq 0$.

In the following sections, we will introduce some results by using CFPDP method.

\subsubsection{Pure mode III (anti-plane shear) crack}

Substituting $K_{\mathrm{I}}=K_{\mathrm{II}}=0, K_{\mathrm{III}} \neq 0$ into equation (9), we can obtain analytic solutions of initial fracture surface. The maximal principal stress is $\sigma_{1}=$ $K_{\text {III }} / \sqrt{2 \pi r}$, the first principal direction is $\boldsymbol{N}_{1}=$ $\sqrt{2} / 2(\sin \theta / 2,-\cos \theta / 2,-1)$, and the initial fracture surface $\boldsymbol{R}_{P}$ is

$$
\begin{array}{r}
\boldsymbol{R}_{P}=r\left(\cos \theta, \sin \theta,-\sin \frac{\theta}{2}\right) \\
\left(0<r<r_{\mathrm{m}},-\pi \leq \theta \leq+\pi\right) .
\end{array}
$$

This is a wide angle cone (Figure 13a). The edge of $r$ is located, where $K_{\text {IIIc }}=K_{\text {Ic }}, K_{\text {Ic }}$ is the toughness of the medium, $\sigma_{\mathrm{m}}$ is the strength of anti-tensile of the medium, so $r_{0}$ is a constant. There are two intersect lines between the fracture surface and the original crack planes. The angles between the two lines and the $x$-axis are $\pm 135^{\circ}$ respectively (Figure $13 \mathrm{a}$ ). The above results
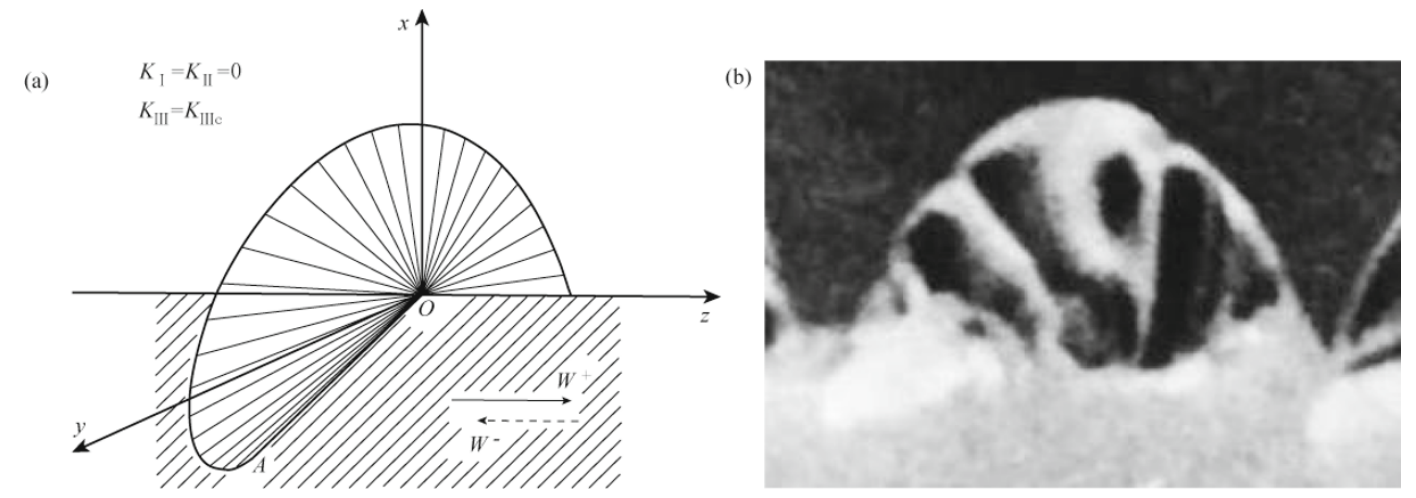

Figure 13 Initial fracture surface of pure mode III crack. (a) Theoretical simulation. (b) Observation of Experiment (Knauss, 1970). 
confirm the experimental results of Knauss (1970) (Figure $13 \mathrm{~b}$ ) quite well. It can be tested and verified that these results are all equal to the results by using the vector methods.

\subsubsection{Mode I (tensile) and mode III (anti-plane} shear) crack

Substituting $K_{\mathrm{II}}=0, K_{\mathrm{I}} K_{\mathrm{III}} \neq 0$ into equation (9), we can find the numerical computing results of the initial fracture surface exhibit symmetrical distortion. The larger is the ratio $K_{\mathrm{I}} / K_{\mathrm{III}}$, the flatter, the longer and the narrower the initial fracture surface becomes. The results of numerical calculation are showed in Figure $14 \mathrm{a}$, which is drawn by adjusting the view angle. A great improvement is achieved compared with the results of Li (1990, 1991). The initial extension shape like double lances is observed as in the experiment of Sommer (1969) (Figure 14b).
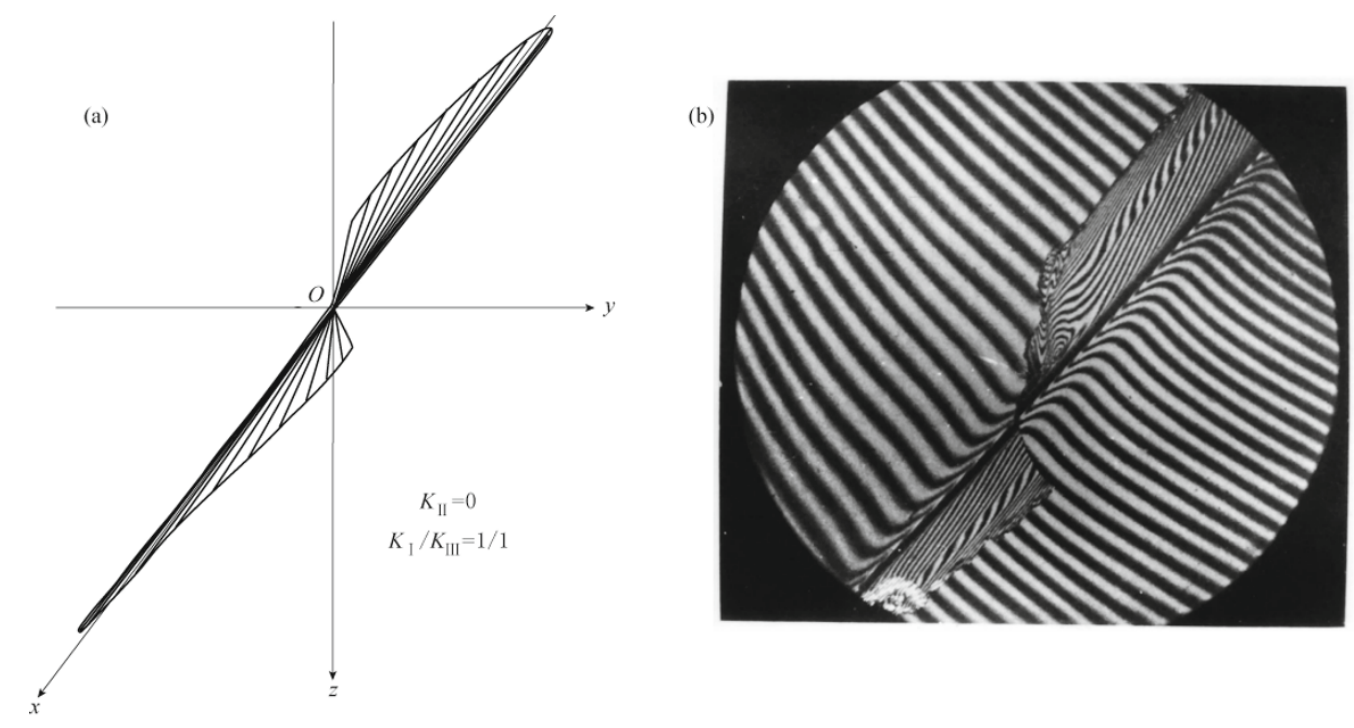

Figure 14 Initial fracture surface of mode I and mode III crack (Poisson ratio $\nu=0.25$ ). (a) Theoretical imitation (Li, 2009); (b) Observation of experiment (Sommer, 1969).

3.3.4 Mode II (in-plane shear) and mode III (Anti-plane shear) crack

Substituting $K_{\mathrm{I}}=0, K_{\mathrm{II}} K_{\mathrm{III}} \neq 0$ into equation (9), we can find the numerical computing results of the initial fracture surface show asymmetrical distort with respect to $x$-z-plane. $r_{\mathrm{m}}(\theta)$ has one maximum at $\theta=-180^{\circ}$ and a minimum at $\theta=+180^{\circ}$ (Figures $15 \mathrm{a}$ and $15 \mathrm{c}$ ), which is consistent with the experimental results (see section 2.3 and Figure 15b) quite well.

Figure 15a corresponds to the places near the crack tip of specimen $\left(\Omega \approx 80^{\circ}\right)$ when $K_{\mathrm{II}} / K_{\mathrm{III}}=1$, and Figure $15 \mathrm{~b}$ corresponds to the places near $\Omega \approx 80^{\circ}$ when $K_{\mathrm{II}} / K_{\mathrm{III}}=1 / 5$. The extended length of asymmetric distortion for the initial fracture has a positive relation with the ratio of $K_{\text {II }} / K_{\text {III }}$.

\subsubsection{Mode I (tensile) and mode II (in-plane shear) crack}

Substituting $K_{\mathrm{III}}=0, K_{\mathrm{I}} K_{\mathrm{II}} \neq 0$ into equation (9), and transforming it into the polar coordinate system, we have

$$
\left\{\begin{array}{l}
\sigma_{r r}=\frac{1}{2 \sqrt{2 \pi r}}\left[K_{\mathrm{I}}(3-\cos \theta) \cdot \cos \frac{\theta}{2}+K_{\mathrm{II}}(3 \cos \theta-1) \cdot \sin \frac{\theta}{2}+o\left(r^{-1 / 2}\right)\right] \\
\sigma_{\theta \theta}=\frac{1}{2 \sqrt{2 \pi r}} \cos \frac{\theta}{2}\left[K_{\mathrm{I}}(1+\cos \theta)-3 K_{\mathrm{II}} \sin \theta+o\left(r^{-1 / 2}\right)\right] \\
\tau_{r \theta}=\frac{1}{2 \sqrt{2 \pi r}} \cos \frac{\theta}{2}\left[K_{\mathrm{I}} \sin \theta+K_{\mathrm{II}}(3 \cos \theta-1)+o\left(r^{-1 / 2}\right)\right]
\end{array}\right.
$$



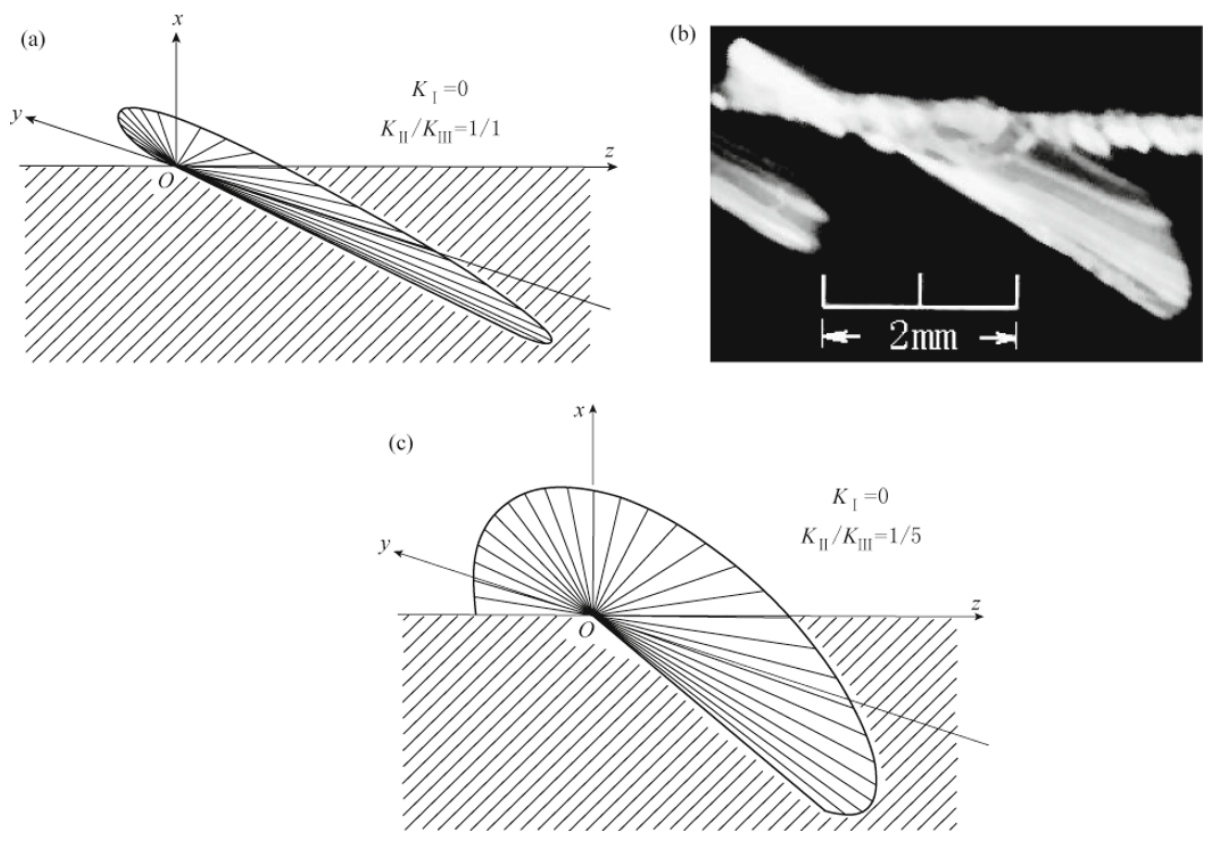

Figure 15 Initial fracture surface of mode II and mode III crack. (a) Theoretical simulation, $K_{\mathrm{II}} / K_{\mathrm{III}}=1$; (b) Observation of experiment; (c) Theoretical simulation, $K_{\mathrm{II}} / K_{\mathrm{III}}=1 / 5$.

According to tensile stress criterion in polar system, $\sigma_{\theta \theta}$ should be principal stress, i.e., $\tau_{r \theta}=0$. If only the single term of stress is kept, the fracture angle is

$$
\theta_{0}=-2 \arctan \frac{\sqrt{1+8\left(K_{\mathrm{II}} / K_{\mathrm{I}}\right)^{2}}-1}{4 K_{\mathrm{II}} / K_{\mathrm{I}}} .
$$

For mode II crack, $\theta_{0}=\arccos (1 / 3)=-70^{\circ} 32^{\prime}$ (Erdogan and Sih, 1963). By using the above results, one can interpret the experimental observation of contact of cylindrical steel indenter with the block of glass (Roesler, 1956). It can be seen that for the force of the indenter edge to the surface of glass, the direction of displacement of inner part of micro-arc line segment is down, i.e., perpendicular to the surface of the specimen. In such way, the fracture is mode II. The axis symmetry makes the shape of extending route become circular cone. The angle between generatrix and the normal direction is just the fracture angle $\theta_{0}$. The angle from the experimental observation is $\theta_{0} \approx-68.5^{\circ}$ which has a $2^{\circ}$ deviation from the theoretical results. We think that the reasons for the deviation are ways of loading and some terms neglected in theoretical resolution.

\subsection{Fractal construction of 3-D fractures}

If the edge of crack near point $O$ is flat, generally, the fracture surface extending from point $O^{\prime}\left(0,0, z_{0}\right)$ is

$$
\boldsymbol{R}_{P}^{\prime}=\left\{r \cos \theta, r \sin \theta, z_{0}-\frac{r\left(l_{0} \cos \theta+m_{0} \sin \theta\right)}{n_{0}}\right\} .
$$

Comparing equation (26) with equation (20), $\boldsymbol{R}_{P}^{\prime}$ is just $\boldsymbol{R}_{P}$ moving $z_{0}$ from point- $O$. This means that the shape of $\boldsymbol{R}_{P}^{\prime}$ is the same as that of $\boldsymbol{R}_{P}$. As $z_{0}$ is arbitrary, any point at the edge of the original crack can become starting point of extension. In such way, infinite initial fracture surfaces can be generated with the same shape. These surfaces are coalesced and restricted each other, which results in finite initial fracture surfaces in finite length along original crack edge.

The observation of experiments in glass shows that the group of the same mode of 3-D fractures generated from the edge of crack exhibit self-similar characteristic. The number of fractal dimension is 1.0, which is deduced as follows.

Let's denote the characteristic scale of the fracture at a level $i$ as $r_{i}$. According to the definition of fractal (Xie, 1993), the number of fracture with characteristic scale $r_{i}$ is $N_{i}=c / r_{i}^{D}$, where $c$ is constant, $D$ is the number of fractal dimension. Thus at level $j$, the number of fracture with the characteristic scale $r_{j}$ is $N_{j}=c / r_{j}^{D}$.

$$
\frac{N_{i}}{N_{j}}=\left(\frac{r_{j}}{r_{i}}\right)^{D}
$$

On the other hand, the experiment results show 
that the distance $d$ of the fractures is positively proportional to $r$, i.e., $d \propto r$, so, $N=L / d \propto L / r$. In such way, $N_{i} \propto L / r_{i}, N_{j} \propto L / r_{j}$. Thus, we have $N_{i} / N_{j}=r_{j} / r_{i}$. Comparing this with equation (27), we get $d=1.0$ at once. Noting that here 1.0 means fraction dimension, which is different from the meaning of ordinary 1 dimension. The latter one is integer.

The fractal construction of $3-\mathrm{D}$ fractures shows that the multiple fractures may occur in different scales, which can be as large as hundreds of kilometer of faults, or as small as the millimeter scale of the crystals of the rock. Superposition of the multiple fractures with the different scales forms the great and small break rockbody, rock mass and fault gauge that are often be seen.

\section{Other application of 3-D frac- ture to faulting}

\subsection{Fracture of an ellipse crack acted by ho- mogenous shear stress}

The slipping section between the asperities in the buried fault plane is often modeled as ellipse crack in an infinite body. Under the pressure in the crust, the shear stress in the fault plane may be treated as nearly homogenous. Thus the initial extensions at the edge of ellipse crack may be calculated as 3-D fracture of modes II and III.

Denote the length of half major axis of the ellipse as $a$, the half minor axis as $b$, the homogenous shear stress acted on the crack plane as $\tau$, and the angle between the direction of $\tau$ and the major axis of the ellipse as $\gamma$ (Figure 16). According to China Academy of Aeronautics (1993), the stress intensity factors at point $A$ with coordinate $x=a \cos \theta, y=b \sin \theta$ at the edge of the ellipse are

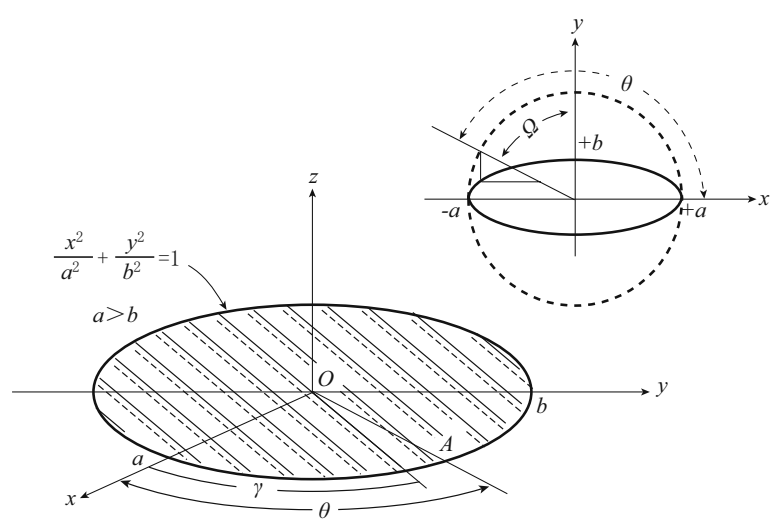

Figure 16 An ellipse crack acted by homogenous shear stress.

$$
\begin{gathered}
K_{\mathrm{II}}(\theta)=\frac{\tau \sqrt{\pi b} k^{2}}{\Theta}\left(\frac{1}{C} \sin \gamma \sin \theta+\frac{k^{\prime}}{B} \cos \gamma \cos \theta\right) \\
K_{\mathrm{III}}(\theta)=\frac{(1-\nu) \tau \sqrt{\pi b} k^{2}}{\Theta} \\
\left(-\frac{1}{B} \cos \gamma \sin \theta+\frac{k^{\prime}}{C} \sin \gamma \cos \theta\right)
\end{gathered}
$$

where $\nu$ is Poisson ratio,

$$
\begin{gathered}
\Theta=\left(\sin ^{2} \theta+\frac{b^{2}}{a^{2}} \cos ^{2} \theta\right)^{1 / 4}, \\
B=\left(k^{2}-\nu\right) E(k)+\nu k^{\prime 2} K(k), \\
C=\left(k^{2}+\nu k^{\prime 2}\right) E(k)-\nu k^{2} K(k), \\
k^{2}=1-\frac{b^{2}}{a^{2}}, \quad k^{2}=\frac{b^{2}}{a^{2}} .
\end{gathered}
$$

$K(k)$ and $E(k)$ are the 1st kind and 2nd kind total ellipse integral, respectively, $E(k)$ is shown in equation (4),

$$
K(k)=\int_{0}^{\pi / 2} \frac{\mathrm{d} \phi}{\sqrt{1-k^{2} \sin ^{2} \phi}} .
$$

Especially when $\gamma=0$, the direction of $\tau$ is parallel to $x$-axis. For convenience to compare with Figure 6 , we denote $\theta=90^{\circ}+\Omega$. Thus the stress intensity factors can be simplified as

$$
K_{\mathrm{II}}=\left(\frac{\pi b^{3}}{a}\right)^{1 / 2} \frac{k^{2} \tau \sin \Omega}{B\left(a^{2} \cos ^{2} \Omega+b^{2} \sin ^{2} \Omega\right)^{1 / 4}}
$$

and

$$
K_{\mathrm{III}}=(\pi a b)^{1 / 2} \frac{(1-\nu) k^{2} \tau \cos \Omega}{B\left(a^{2} \cos ^{2} \Omega+b^{2} \sin ^{2} \Omega\right)^{1 / 4}},
$$

then

$$
\frac{K_{\mathrm{II}}}{K_{\mathrm{III}}}=\frac{b \tan \Omega}{a(1-\nu)}
$$

It is obvious that for any value of $\Omega$, the value of $K_{\mathrm{II}}$, $K_{\text {III }}$ and the ratio $K_{\text {II }} / K_{\text {III }}$ can be determined, and the shape of initial fracture surfaces can be calculated by using the method of principal planes in this paper. These theoretical solutions of different ratios $K_{\mathrm{II}} / K_{\mathrm{III}}$ refer to section 3.3.4. The whole shape of fracture distribution in the area $-90^{\circ} \leq \Omega \leq 90^{\circ}$ with different normal stresses is similar to the experimental results shown in Figure 8. For example, at $\Omega= \pm 90^{\circ}, K_{\mathrm{III}}=0$, the fracture belongs to mode II,

$$
K_{\mathrm{II}}=\left(\frac{\pi}{a}\right)^{1 / 2} \frac{b k^{2} \tau}{B} \quad\left(\Omega= \pm 90^{\circ}\right) .
$$


At $\Omega=0$, the fracture belongs to mode III,

$$
K_{\mathrm{III}}=(\pi b)^{1 / 2} \frac{-(1-\nu) k^{2} \tau}{B} \quad\left(\Omega=0^{\circ}\right) .
$$

From the above equations, it is obvious that under the condition of $a \gg b$, we have $\left|K_{\mathrm{II}}\right|_{\Omega= \pm 90^{\circ}} \ll\left|K_{\mathrm{III}}\right|_{\Omega=0^{\circ}}$. In such way, the critical loading values are different in different points at the crack edge. At the point of $\Omega=0$, the critical condition is approached firstly, and the pure mode III fracture is initiated. Then at the points $0^{\circ}<|\Omega|<90^{\circ}$, the fractures with different ratio of $K_{\mathrm{II}} / K_{\mathrm{III}}$ are initiated one by one. At the point $\Omega= \pm 90^{\circ}$ the critical condition is approached to at last, and mode II fracture is initiated. Here the extensions are the longest and called main fracture. In such way, there are no essential differences of the fractures between the half section of ellipse crack, i.e., $-90^{\circ} \leq \Omega \leq+90^{\circ}$, with the surface crack shaped semi-ellipse, just only some difference in the quantity of ratio of $K_{\text {II }} / K_{\text {III }}$. The distribution in the section of $90^{\circ}<\Omega<270^{\circ}$ and the section of $-90^{\circ}<\Omega<+90^{\circ}$ are symmetric.

These theoretical solutions can verify the guess of 3-D fracture mode of fault proposed by Scholz (1990).

\subsection{EDA model of anisotropy of medium}

From the fractures of modes II and III of 3-D crack under pressure, it is shown that the directions of initial extensions are the same roughly. This phenomenon called directional arrangement of fissures. Extensive Dilatancy Anisotropy (EDA), is the important mechanism of anisotropy caused by evolution of micro-cracks, and important theoretical basis of precursors of $\mathrm{S}$ wave splitting and earth resistivity.

\subsection{Tectonophysics}

The 3-D structure of fault is the important study object of tectonophysics. For instance, the en echelon array faults in the ground surface, the tensile fractures form tension basin together with filling (Atkinson, 1987). The 3-D propagation of fault is described as "obviously bending, simply and independent propagation of small shear fault", and "cone or helical shear fracture" (Gezuofusiki, 1975), as shown in Figure 17. The above description of tensile fissures can be interpreted as 3-D fracture of anti-plane shear fault.

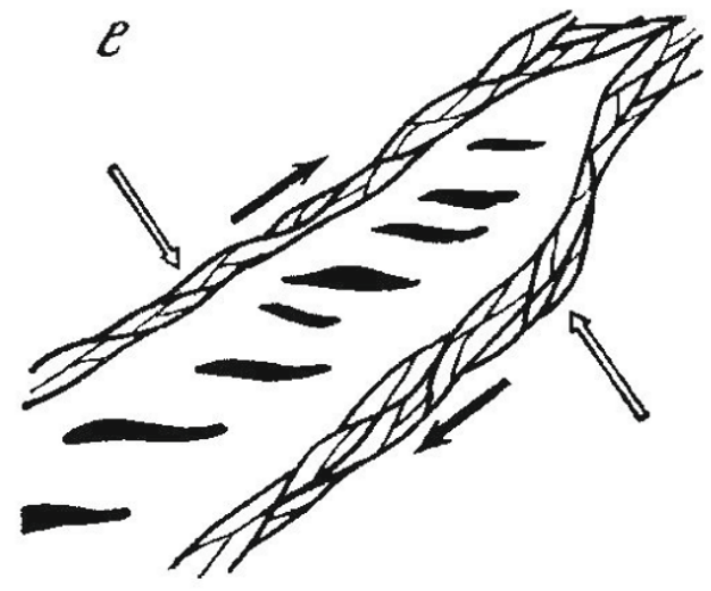

Figure 17 En echelon array of tensile fissures of shear fault (Gezuofusiki, 1975), where the black arrows indicate the direction of fault dislocation.

\subsection{3-D fractures in rock}

Cox and Scholz (1988) observed some 3-D fractures under shear stress in rock specimen by using microscope. Wu (1991) presented a lot of evidences of 3-D fatigue fractures of rock in field and indoor slice of quartz. He proposed that these fatigue fractures are the integral of tidal force action. From theses pictures, we can see that the shapes of rock fatigue fracture are quite similar to the quasi-helical 3-D fractures in the experimental observations.

\subsection{The multiple earthquake events}

The 3-D fracture model can be used to interpret the non-coplanar of the sources distribution of subevents of large earthquake (Tseng and Shi, 1978). If the sub-events do not occur in transient, but one by one in a longer time, they may form foreshock or parts of aftershock. In Figure 18, the 3-D locations of the foreshocks of $M_{\mathrm{S}} 5.6$ Xiuyan-Haicheng earthquake on November 29, 1999 (Zhang et al., 2001) are shown as the series number of time. It is also shown that the five $M_{\mathrm{L}} \geq 4$ foreshocks occurred at the outer edge of a helical surface, and the main shock is located in the inner edge. The shape of the helical is just similar to the theoretical result and the experimental observation of pure mode III fracture. 

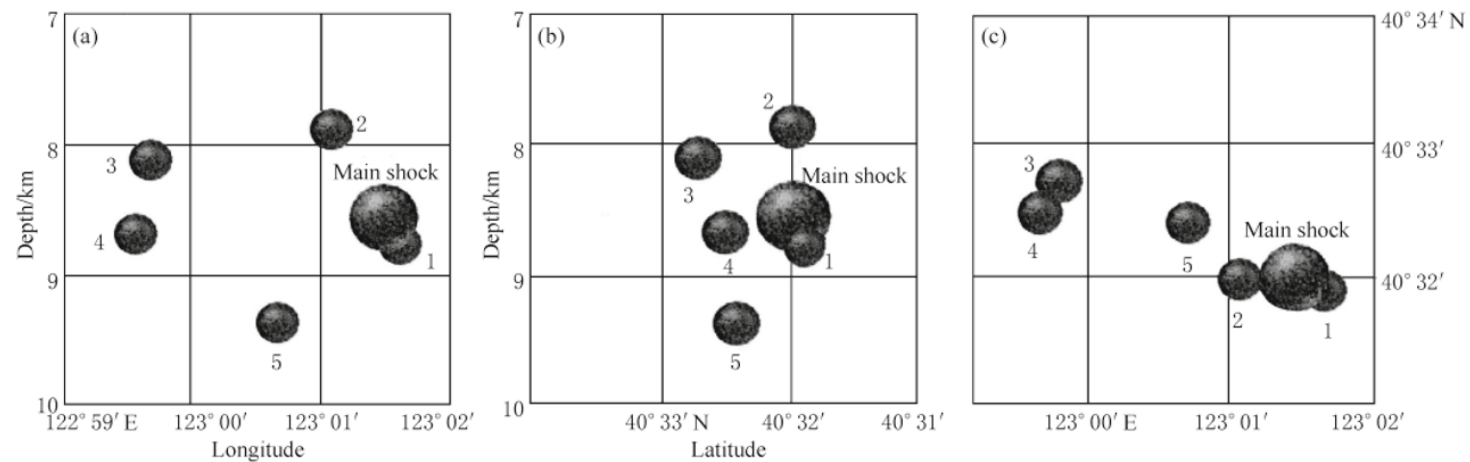

Figure 18 The 3-D locations of the fore-shocks of $M_{\mathrm{S}} 5.6$ Xiuyan-Haicheng earthquake on November 29, 1999 (Zhang et al., 2001). (a) Figure of latitude profile (master view). (b) Figure of longitude profile (left view). (c) Figure of platform.

Acknowledgements This study is supported by National Natural Science Foundation of China (No. 41090292) and by the special professional term 8-54 of China Earthquake Administration.

\section{References}

Atkinson B K (1987). Fracture Mechanics of Rock. Academic Press Limited, London, 557pp.

China Academy of Aeronautics (1981). Handbook of Stress Intensity Factors. Science Press, Beijing, 384-388 (in Chinese)

China Academy of Aeronautics (1993). Handbook of Stress Intensity Factors (Suppl). Science Press, Beijing, 651654, 728-731 (in Chinese).

Cox S J D and Scholz C H (1988). On the formation and growth of faults: An experimental study. Journal of Structure Geology 10: 413-30.

Ding Z F, Wang C Y, Zhou M D, Winston Chan, You H C, He C S, Wu J P and Chang Z G (2004). Shallow seismic exploration on the rupture of the $M_{\mathrm{S}} 8.1$ Kunlun earthquake. In: Chen Y T, Kan R J, Wang C Y eds. Advances in Continental Seismology and Physics of Earth's Interior in China: In Honor of Academician Zeng Rongsheng's 80th Birthday. Seismological Press, Beijing, 122-127 (in Chinese).

Erdogan F and Sih G C (1963). On the crack extension in plates under plane loading and transverse shear. $J$ Basic Engng 85: 519-527.

Fan L S, Jia J, Wu J S, Zhao Y G and You J W (2009). Overview on drilling operation of the (WFSD-1) in Wenchuan fault scientific drilling project. Exploration Engineering 36(12): 5-8 (in Chinese with English abstract).

Gezuofusiki (1975). Foundamentals of Tectonophysics. Science Press, Moscow, 535pp (in Russian).

Knauss W G (1970). An observation of crack propagation in anti-plane shear. Int J Frac 6: 183-187.
Lawn B R and Wilshaw B R (1975). Fracture of Brittle Solids. Cambridge Press, Cambridge, 204pp.

Li S Y (1990). Normal stress criterion of a three-dimensional brittle fracture. Chinese J Geophys 33(3): 353-363 (in Chinese with English abstract).

Li S Y (1991). Spontaneous Propagation of In-plane Shear Fault. [Ph.D. Dissertation]. Institute of Geophysics, State Seismological Bureau, 148pp (in Chinese with English abstract).

Li S Y, Teng C K, Liu X H and He X S (2000). Experimental investigation in rock rupture with the co-operation between Chinese and Russian seismologists. Recent Developments in World Seismology (3): 1-3 (in Chinese with English abstract).

Li S Y, He T M and Yin X C (2009). Introduction to Rock Fracture Mechanics. University of Science and Technology Press, Hefei, 468pp (in Chinese).

Palaniswamy K and Knauss W G (1978). On the problem of crack extension in brittle solids under general loading. In: Nemat-Nasser ed. Mechanics Today. Pergamon Press Inc., New York, 4: 87-148.

Qian W C and Ye K Y (1956). Elastic Mechanics. Science Press, Beijing, 422pp (in Chinese).

Roesler F C (1956). Brittle fracture near equilibrium. Proc Phys Soc B69: 981-992.

Ruff L J (2002). State of stress within the Earth. In: William H K Lee, Hiroo Kanamori and Paul C Jenning eds. International Handbook of Earthquake and Engineering Seismology. 81A. Academic Press for International Association of Seismology and Physics of the Earth's Interior, Amsterdam, 539pp.

Scholz C H (1990). The Mechanics of Earthquakes and Faulting. Cambridge University Press, London, 439pp.

Sommer E (1969). Formation of fracture lances in glass. Engineering Fracture Mechanics 1: 539-546.

Teng C K, Yin X C, Li S Y and Cai D E (1987). Experimental study of three dimensional fracture in plane specimens with non-penetrating crack. Acta Geo- 
physica Sinica 30(4): 371-378 (in Chinese with English abstract).

Tian J, Zhu Z J, Zhu Z D, Yao Y X and Wang Y (2009). Discussion of seismic velocity anomalies of Longmenshan fault zone after Wenchuan 8.0 earthquake. Plateau Earthquake Research 4: 21-24 (in Chinese with English abstract).

Timoshenko S P and Goodier J N (1970). Theory of Elasticity. 3rd edition. McGraw-Hill Co., New York, 567pp.

Tseng J S and Shi J S (1978). Source Multiplicity of the main shock of the Yongshan-Daguan Yunnan earthquake of May 10, 1974. Chinese J Geophys 21: 160-173 (in Chinese with English abstract).

Wu H W (1991). Fatigue fracture under the cumulate damageone of the basic mechanisms for structural deformation of the crust. Scientia Geologica Sinica 2: 101-110 (in Chinese with English abstract).

Xie H (1993). Fractals in Rock Mechanics. A A Balkema Publishers, Rotterdam, 369pp (in Chinese).

Yin X C, Li S Y, Li H and Wang M (1988). Experimental study of interactions between two flanks of a closed crack. Acta Geophysica Sinica 31(3): 306-314 (in Chinese with English abstract).

Yin X C, Li S Y, Teng C K, Li H and Li J (1989). An experimental investigation on the extension of non-penetrating cracks in rocks and other brittle solids. Earthquake Research in China 3(1): 1-11 (in Chinese with English abstract).

Yin X C, Li S Y, Li H, Teng C K and Li J (1991). An experimental investigation on extension of non-penetrating cracks in rocks and other brittle solids. The 17th International Conference of Theoretical and Applied Mechanics 1988, Grenoble France. Peking University Press, Beijing, 156-166 (in Chinese).

Zhang Q, Pan K, Zhan H Y and Wang S H (2001). The study on the characteristics of spatial distribution for the Xiuyan-Haicheng earthquake sequence $\left(M_{\mathrm{S}}=5.4\right)$. Seismological and Geomagnetic Observation and Research 22(2): 83-91 (in Chinese with English abstract). 